\title{
External Truthfulness
}

\begin{abstract}
This chapter proposes some analytical tools for understanding how communication in general and narration in particular can be truthful to what one perceives to be the actual world; how it can achieve external truthfulness. These external connections are scrutinized in terms of various sorts of contiguity, forming the basis for indices that connect narratives to the perceived actual world. The proposed analytical tools are intended to make it possible to understand the many ways in which the represented events in narratives can be connected to phenomena outside the narratives. The standard concept for theorizing this issue within narratology - fictionality - is critiqued and replaced with a multifaceted concept of (lacking) external truthfulness.
\end{abstract}

Keywords Transmedial narration $\bullet$ Truthfulness $\bullet$ Contiguity $\bullet$ Index - Fiction • Fictionality

In Chap. 8, I circumscribed narration in terms of intracommunicational indexicality creating internal coherence. To conclude this second part of the treatise, I will also investigate narration in the light of extracommunicational indexicality forming external truthfulness. As internal coherence, external truthfulness is a concept that is potentially valid for all kinds of virtual spheres created in communication. Traditionally, however, and for good reasons, issues of truthfulness have often been connected to research

(C) The Author(s) 2019

L. Elleström, Transmedial Narration, https://doi.org/10.1007/978-3-030-01294-6_9 
on narration. When perceiving a narrative, nothing is more natural than to ask whether the story is 'true' or not; do the core events of the narrative correspond to events outside the narrative? The standard way of theorizing this issue within narratology is through the concept of fictionality. In this chapter, for reasons that will become clear, I will instead elaborate on the concept of extracommunicational indexicality.

Extracommunicational indexicality is semiosis that creates bonds between a virtual sphere and its surroundings, connecting representamens on the ground of contiguity to objects from outside the virtual sphere. I suggest this is external truthfulness in communication. Thus, the concept of truthfulness that I propose is to be understood as a conceived communicative trait; this is not to be confused with truth, which is understood as a feature of the actual, never fully accessible world. However, truth may possibly be approached through accumulated truthful communication and the observation of effects of further action on the basis of conceived truthfulness-if the effects of the actions correspond to what is predicted by the communication, there is a chance that truthfulness will come close to truth.

\section{Communicators and Narrators}

Approaching the issue of external truthfulness, one factor deserves special attention: communicating minds, understood as communicators (and more specifically narrators), not communicatees (narratees). As defined in this treatise, communication is about transferring cognitive import among minds. Therefore, the concept of communicator is germane. In related but clearly different ways, communicators are central to conceiving both the intracommunicational and the extracommunicational domain. To a certain extent, they are responsible for both internal (in)coherence and external (un)truthfulness. This comes about through representation. Communicators are made present to the mind of the perceiver, the actual communicatee, via representamens of the media products, and they may be objects in the virtual sphere itself, in other virtual spheres, or in the perceived actual sphere.

The starting point for this inquiry is the plain but fundamental observation that actual communicators, producing some cognitive import to be perceived by actual communicatees via media products, do not simply disappear behind the virtual spheres created in the perceivers' minds. In many situations, the actual communicator is decidedly represented by the 
media product and so becomes part of the virtual sphere. In an ordinary conversation, for instance, the word ' $\mathrm{I}$ ' is often understood as an index, based on strong contiguity in tangible space and time, for the actual communicator using her body and its extensions as media products when uttering the word. To the extent that anything can be established at all, this is a determinable communicating mind that can even be engaged in two-way communication. In other situations, the actual communicator may be much more distant in both space and time and sometimes, such as when one looks at ancient rock-paintings, the actual communicator is not at all accessible and can only be construed as an idea of something that must have existed at some point. The painting becomes an index based on a weak contiguity that depends on the assumption that someone must have produced the visual configurations through actions of mind and hand. In any case, actual communicators are always, if they are parts of the perceived actual sphere, perceived actual producers' minds.

Represented actual communicators, originating in the perceived actual sphere, are objects that warrant external truthfulness, to varying degrees. Their existence in and collateral experiences of certain parts of the perceived actual sphere make it plausible that certain aspects of the communicated cognitive import are more or less truthful, even though, paradoxically, actual communicators in fact become virtual the moment they are represented. Of course, the representation of actual communicators is not in itself a guarantee of complete truthfulness (for instance, there are factors such as forgetfulness, misconceptions, and lies that disconnect parts of the intracommunicational domain from the extracommunicational domain), but the collateral experience of actual communicators makes it possible to partly decide upon the amount of contiguity that is present. This is a complex issue that cannot be developed further for the moment; here, I only want to stress that actual communicators are central extracommunicational objects; although their roles may vary considerably, they are always, at a minimum, necessary links to the perceived actual sphere.

Apart from representing actual communicators, media products may also represent overarching and embedded virtual communicators. Although emerging within the virtual sphere, overarching virtual communicators, like all intracommunicational objects, are ultimately construed by extracommunicational objects, which means that they may well be similar to actual communicators. In addition, media products may represent all kinds of communicators that have already been represented in other virtual spheres (one recognizes the communicators from earlier communica- 
tion); these are extracommunicational objects that can be incorporated in a virtual sphere in intricate ways.

\section{Kinds OF TRUTHFulness}

Although actual communicators are often central for the conception of external truthfulness through their presence in the perceived actual sphere, they do not, as such, determine the outcome of communication. Ultimately, it is the actual communicatees that perceive media products and form virtual spheres on the grounds of specific media traits and surrounding factors. More precisely, it is those media traits that are perceived to have real connections to the extracommunicational domain, and furthermore trigger indexical interpretation, that ultimately create external truthfulness.

One central question that has been largely missing in narratological research, and more broadly in communication research, is: To what exactly can communication, and therefore narration, be truthful? Only differentiating different kinds of external truthfulness can help us out of the trap of such unproductive dualities as truthful versus untruthful and fiction versus nonfiction, which too often lead to either-or ways of reasoning.

Therefore, I suggest that extracommunicational indexical objectsobjects from the extracommunicational domain that are represented on the ground of real connections - can be classified variously, each category corresponding to a certain kind of truthfulness. Here, I will provide some prominent examples of such kinds of objects and truthfulness. It is not a rigid classification but rather an incomplete inventory of types that sometimes overlap, sometimes complement each other, and sometimes are in conflict. I do not propose that they should be treated as categories for compartmentalization; rather, they are flexible groupings for methodical investigations of truthfulness in communication.

Following the division of the extracommunicational domain into two parts, we may state that a virtual sphere can be truthful to objects in the perceived actual sphere or to objects in other virtual spheres; to our notions of the surrounding world or to our acquaintance with earlier communication. In turn, earlier communication may be truthful to objects in the perceived actual sphere or to objects in other virtual spheres. The notion that there may be truthful representations of other virtual spheres that do not represent the perceived actual sphere has previously been 
discussed in terms of making truthful performances and statements about so-called fictional characters (Colapietro 2009: 117; Searle 1975: 329).

Another division that follows from our earlier discussions in this treatise is that a virtual sphere can be truthful to objects that are material or mental. This is a crucial and, in a way, self-evident, but often neglected observation. According to my view, a concept of truthfulness that includes only real connections to materially observable states is perhaps easier to manage, but of little use.

Connecting to an age-old distinction of Aristotle, we can also say that a virtual sphere can be truthful to objects that are (more or less) universal or those that are particular (Aristotle 1997 [c. 330 BCE]: 81; cf. Gale 1971: 335; Gallagher 2006: 341-343; Walton 1983: 80). Some variations of this distinction would be to say that a virtual sphere can be truthful to objects that are typical or atypical; permanent or temporary; and global or local. This could perhaps be understood as a sort of statistical view on truthfulness, related to the probability of repeated contiguity in various environments and circumstances; truthfulness as a function of certain ways of framing the extracommunicational domain.

In a related manner, a virtual sphere can be truthful to objects that are wholes or to objects that are details (cf. Pavel 1986: 17). Truthfulness in detail does not guarantee a truthful whole and a truthful whole may harbor non-truthful details. This is truthfulness understood as perception of gestalts; truthfulness emanating from (in)attention to (absence of) singular real connections when construing the overall pattern of contiguity.

An important but more complex way of sorting extracommunicational indexical objects, partly coinciding with some of the earlier categories, is that a virtual sphere can be truthful to objects that have previously been manifested, that are currently manifested, that are bound to be manifested, or that may be manifested (cf. the concept of "possible worlds" in Pavel 1986: 46). One could perhaps even argue that a virtual sphere can be truthful to objects that should be manifested. These latter kinds of truthfulness rely heavily on mental contiguity.

In this context, it must also be noted that any material item can be drawn into the communicative act and become a media product working together with other media products or creating highly multimodal joint media products. In criminal trials, for instance, fingerprints and other pieces of evidence are framed so that they interact with standard basic media types such as speech, written text, still images, movies, and sound 
recordings that incorporate them to create a virtual sphere based on strong contiguity to the perceived actual sphere.

Summarizing these recent observations, and some earlier ones from Chap. 8, I suggest that all general varieties of contiguity (from weak copresence to strong interaction), all kinds of indexical junctions (based on mechanical, electromagnetic, chemical, organic, and mental contiguity), and all types of indexical objects (in the perceived actual sphere or in other virtual spheres, material or mental, universal or particular, wholes or details, manifested previously, currently, or subsequently) are involved in claims to external truthfulness in communication. Consequently, they are all vital to external truthfulness in narration.

\section{Truthfulness in So-Called Fiction}

To close this chapter, and also Part II of the treatise, I will place external truthfulness in relation to the contrasting concepts of fiction and fictionality. Fictionality is normally understood as a supposed (at least partial) quality of certain qualified media types labeled fiction- "novel, short story, graphic novel, fiction film, television serial fiction, and so on" (Skov Nielsen et al. 2015: 62; cf. Searle 1975: 332)—and pertaining to representation of invented, unreal, and purely imaginary objects. Also, when fictionality is sometimes assumed to be a possible quality in nonfiction, it is circumscribed in terms of invention and unreality.

In other words, fictionality is supposedly not the representation of objects from the perceived actual sphere but solely of objects from the virtual sphere or other virtual spheres that do not involve the perceived actual sphere. This concept runs into trouble when one considers that all intracommunicational objects ultimately rely on extracommunicational objects, even though they emerge within the intracommunicational domain and may gain a sort of autonomy by being perceived as new gestalts. A minimal conclusion of this observation is that fictionality is very difficult to circumscribe because of the floating borders between extracommunicational objects and new intracommunicational gestalts. A more drastic conclusion is that there is no specific quality of fictionality, only sorts and degrees of truthfulness according to the categorizations in the last sections: degrees of contiguity from weak co-presence to strong interaction in various indexical junctions (based on mechanical, electromagnetic, chemical, organic, or mental contiguity) connecting to a variety of indexical objects (in the perceived actual sphere or in other virtual spheres, 
material or mental, universal or particular, wholes or details, manifested previously, currently, or subsequently). In less provocative terms, this would mean that the idea of fictionality is not necessarily meaningless or redundant but in dire need of a refined conception of the myriad ways in which communication can harbor low degrees of truthfulness.

If the term 'fictionality' is to be retained at all, it should not be understood as referring to distinct features but to a lack of certain sorts of truthfulness. In effect, this renders the term 'fictionality' superfluous. Hence, I argue that truthfulness and so-called fictionality are not two contrary qualities; rather, they represent different grades on the same scale-and one does not need two terms, and even less two concepts, to capture the variations of one phenomenon. I think it is more efficient to work with one homogeneous but indeed very complex concept of sorts and degrees of (lack of) truthfulness.

If the concept of fictionality is deserted or remodeled to a notion of lacking truthfulness, what is then left of fiction, which is supposedly based on fictionality? Under all circumstances, it is clear that one cannot make "a categorical distinction" between fiction and nonfiction (Yadav 2010: 191; cf. Ryan 1991). If the concept of nonfiction is to be retained in academic discourse, it must be understood as a range of qualified media types that are expected to have certain kinds of truthfulness. Fiction, an equally problematic concept, would then be a range of qualified media types that are expected to lack certain kinds of truthfulness. However, this does not eliminate the condition that there is truthfulness in both fiction (including qualified media types such as novels, animated cartoons, and ballads) and nonfiction (such as documentary films, scientific articles, and oral testimonies). This has been acknowledged in various ways by several scholars who otherwise differ in their conceptions and terminology (for instance, D'Alessandro 2016; Gale 1971; Grishakova 2008; Harshaw 1984; Ronen 1988; Ryan 1980; Searle 1975).

Because I find this conception of fiction versus nonfiction very coarse and unnecessarily cumbersome, I think it gives a better understanding of the varieties of truthfulness in communication if each qualified media type is investigated on a more fine-grained scale regarding expected truthfulness in terms of different kinds of contiguity and different kinds of extracommunicational indexical objects. I will illustrate this with some observations of a few qualified media types from the historical and cultural perspective in which the author of this treatise is situated. 
Television news programs are normally expected to be strongly truthful in a variety of ways. They should preferably include photographs or film footage produced by electromagnetic or chemical contiguity. They should certainly be truthful to objects in the perceived actual sphere, but also to objects in other virtual spheres, meaning that earlier communication must be correctly reported. News programs should also have real connections to both material and mental objects; not only to persons, places, and events but also to objects such as ideas and emotions. Both wholes and details are expected to appear correctly. Importantly, these programs are expected to truthfully represent objects that are particular, regardless of their degree of universality, which means that atypical and temporary rather than permanent objects are also part of their norm. Furthermore, the programs should definitely be equally truthful to objects that have been manifested and those that are currently manifested-and, if possible, to objects that may or are bound to be manifested.

In contrast, historical paintings are expected to be strongly truthful in some ways and less truthful in others. To be counted as part of this qualified media type, a media product should be produced by mental and mechanical contiguity by a person possessing relevant collateral experience. A historical painting ought to be truthful to mainly material, visual objects. Although the quality of universality can certainly be included, it is primarily expected to have real connections to objects particular to a certain time and place. It is foreseen to be truthful to both wholes and details, although the very smallest details are often counted out. While the primary norm is to truthfully represent objects that have been manifested, this might well be combined with truthful representation of objects that may be manifested according to the idea that history can repeat itself.

A third example is science fiction novels that are expected to be more or less truthful in other ways compared to news reports and historical paintings. To a certain extent, they should be truthful to objects in other virtual spheres, meaning that their own objects should preferably correspond to other science fiction in order to make sense. Most readers probably anticipate such novels to represent more or less universal objects, and to discuss things in general and globally. Of course, this does not exclude truthful representations of atypical and spectacular objects. Naturally, science fiction novels are primarily expected to be truthful to objects that may be, and perhaps to some extent ought or ought not to be, manifested in the future. 
My claim here is not that the sketched expectations of a handful of qualified media types are accurate, but rather that there are various and shifting anticipations of these kinds that are important for construing qualified media types. Media types and submedia, or genres, are often qualified (Elleström 2010) exactly regarding expected presence or absence of various sorts of truthfulness; qualified media types are partially defined by the very kinds of truthfulness in the media products that constitute them (cf. Wildekamp et al. 1980: 556). Thus, media type attributions such as 'this is a dinner conversation, but that is a legal testimony' can be understood as truth claims. Additionally, expected or even required varieties of external truthfulness and non-truthfulness are often envisaged to go handin-hand with certain styles and other media hallmarks that emphasize the media differences. In the end, however, qualified media types are certainly not stable entities but important pragmatic categories that vary through history, ideologies, and cultures. Mapping such manifold diversities is necessary in order to transcend the all too coarse fiction-nonfiction distinction.

In this concluding section of the last chapter of the second part of the treatise, I have already moved the discussion from basic to qualified media types. In the third and final part, the qualifying aspects of media types will be studied in some detail and from a decidedly narrative point of view.

\section{REFERENCES}

Aristotle. 1997 [c. 330 BCE]. Aristotle's Poetics, ed. John Baxter and Patrick Atherton. Translated by George Whalley. Montreal: McGill-Queen's University Press.

Colapietro, Vincent. 2009. Pointing things out: Exploring the indexical dimensions of literary texts. In Redefining Literary Semiotics, ed. Harri Veivo, Christina Ljungberg, and Jørgen Dines Johansen, 109-133. Newcastle upon Tyne: Cambridge Scholars Press.

D'Alessandro, William. 2016. Explicitism about truth in fiction. British Journal of Aesthetics 56: 53-65.

Elleström, Lars. 2010. The modalities of media: A model for understanding intermedial relations. In Media Borders, Multimodality and Intermediality, ed. Lars Elleström, 11-48. Basingstoke: Palgrave Macmillan.

Gale, Richard M. 1971. The fictive use of language. Philosophy 46: 324-340.

Gallagher, Catherine. 2006. The rise of fictionality. In The Novel, Vol. 1: History, Geography, and Culture, ed. Franco Moretti, 336-363. Princeton, NJ: Princeton University Press. 
Grishakova, Marina. 2008. Literariness, fictionality, and the theory of possible worlds. In Narrativity, Fictionality, and Literariness: The Narrative Turn and the Study of Literary Fiction, ed. Lars-Åke Skalin, 57-76. Örebro: Örebro University Press.

Harshaw, Benjamin. 1984. Fictionality and fields of reference: Remarks on a theoretical framework. Poetics Today 5: 227-251.

Pavel, Thomas G. 1986. Fictional Worlds. Cambridge, MA: Harvard University Press.

Ronen, Ruth. 1988. Completing the incompleteness of fictional entities. Poetics Today 9: 497-514.

Ryan, Marie-Laure. 1980. Fiction, non-factuals, and the principle of minimal departure. Poetics 9: 403-422.

- 1991. Possible Worlds, Artificial Intelligence, and Narrative Theory. Bloomington and Indianapolis: Indiana University Press.

Searle, John R. 1975. The logical status of fictional discourse. New Literary History 6: 319-332.

Skov Nielsen, Henrik, James Phelan, and Richard Walsh. 2015. Ten theses about fictionality. Narrative 23: 61-73.

Walton, Kendall L. 1983. Fiction, fiction-making, and styles of fictionality. Philosophy and Literature 7: 78-88.

Wildekamp, Ada, Ineke van Montfoort, and Willem van Ruiswijk. 1980. Fictionality and convention. Poetics 9: 547-567.

Yadav, Alok. 2010. Literature, fictiveness, and postcolonial criticism. Novel 43: 189-196.

Open Access This chapter is licensed under the terms of the Creative Commons Attribution 4.0 International License (http://creativecommons.org/licenses/ by $/ 4.0 /$ ), which permits use, sharing, adaptation, distribution and reproduction in any medium or format, as long as you give appropriate credit to the original author(s) and the source, provide a link to the Creative Commons licence and indicate if changes were made.

The images or other third party material in this chapter are included in the chapter's Creative Commons licence, unless indicated otherwise in a credit line to the material. If material is not included in the chapter's Creative Commons licence and your intended use is not permitted by statutory regulation or exceeds the permitted use, you will need to obtain permission directly from the copyright holder.

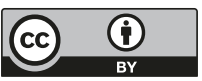

